\title{
Genetic analysis of exopolysaccharide acetilation product by Burkholderia cepacia
}

\author{
Linda Furlanis, Lucia Corich, Francesco Gon, Enrico Angelo Tonin, Lucilla Dolzani, \\ Cristina Lagatolla \\ Dipartimento di Scienze della Vita, Università degli Studi di Trieste
}

Key words: Burkholderia cepacia, Exopolysaccharide, Acyltransferase

Analisi genetica dei livelli di acetilazione dell'esopolisaccaride prodotto da Burkholderia cepacia

\section{SUMMARY}

Bacteria belonging to the Burkholderia cepacia complex are mainly isolated from the sputum of cystic fibrosis patients and frequently show a mucoid phenotype. Most of them produce an exopolysaccharide called cepacian, whose repeating unit consists of a branched heptasaccharide carrying from one to three acetyl esters. Two genetic loci, bcel and bcell, consisting of II and 9 genes respectively, are involved in cepacian biosynthesis. Three genes located in the bcell locus, named bceOSU, code for different acyltransferases. As the presence of acetyl groups influences the viscosity of cepacian, we compared three strains (two clinical isolates named BTS2 and BTS7, and the reference strain Burkholderia sp. 383) exhibiting differences both in the acetylation pattern and at the genomic level, for the presence of insertion sequences adjacent to bceU.

\section{INTRODUZIONE}

Burkholderia cepacia, microrganismo isolato prevalentemente in pazienti affetti da fibrosi cistica, presenta frequentemente fenotipo mucoide.

Il più comune esopolisaccaride (EPS) prodotto è chiamato cepaciano: è costituito da un'unità ripetitiva (UR) eptamerica ramificata (Figura I), acetilata fino a tre volte (1). Per la sintesi di questo EPS sono stati individuati 2 loci genici, bceI e bcell, costituiti rispettivamente da 11 e 9 geni $(2,3)$.

$\mathrm{Nel}$ locus bceII sono localizzati i geni bceOSU, che codificano per tre differenti aciltransferasi.

La presenza di gruppi acetile influenza la viscosità del cepaciano.

Due isolati clinici, BTS2 e BTS7 e il ceppo di riferimento Burkholderia spp 383, il cui genoma è depositato in banca dati, sono stati studiati perché presentano delle differenze a livello dei geni bceOSU cui corrispondono diversi pattern di acetilazione dell'EPS.

\section{MATERIALI E METODI}

Il sequenziamento dei loci degli isolati clinici è stato ottenuto da sub-cloni della regione d'interesse oppure da prodotti di PCR ottenuti con primer specifici. Per la ricostruzione dell'intero locus è stato utilizzato il programma ContigExpress contenuto nella suite Vector NTI Advance 10 (Invitrogen). Per identificare i geni contenuti nella regione di interesse è stato utilizzato il programma ORF finder fornito dall'NCBI. L'analisi dei promotori è stata eseguita con il programma
"BPROM- Promoter finding in Bacteria". Infine è stata identificata la forcina di terminazione dell'operone tramite il programma "FindTerm - Finding Terminators in bacterial genomes".

\section{RISULTATI}

Sono state ricostruite le sequenze dei loci bceI e bceII sia di BTS2 che di BTS7.

L'analisi di sequenza di bcell ha rivelato due regioni aggiuntive in BTS2, omologhe a geni codificanti transposasi (tnp), ma inattive per la presenza di numerosi codoni di stop (Figura II).

Una in particolare è inserita in posizione adiacente al gene codificante l'aciltransferasi BceU. Apparentemente il loro inserimento non determina inattivazione di tali geni, ma l'analisi mediante $1 \mathrm{H}-\mathrm{NMR}$, svolta dal gruppo di ricerca del Prof. Rizzo (DSV) con cui collaboriamo, ha dimostrato che il numero medio degli acetili presenti nell'UR del cepaciano prodotto da BTS2 è pari a 2.2 , contro i 3.0-2.8 presenti in quello prodotto rispettivamente da BTS7 e Burkholderia 383 (Tabella 1).

\section{CONCLUSIONI}

Poiché nell'UR dell'EPS prodotto da Burkholderia 383 e BTS7 sono presenti mediamente 3 acetili mentre in quello prodotto da BTS2 ce ne sono mediamente 2 , è possibile ipotizzare che l'inserzione di uno dei geni tnp abbia determinato l'inattivazione di uno dei geni codificanti per aciltransferasi, la cui espressione verrà studiata in futuro mediante RT-PCR.

\section{Corresponding author: Linda Furlanis}

Dipartimento di Scienze della Vita, Università di Trieste

Via L. Giorgieri, I - 34I00 Trieste - Tel.: 0405583693 - Fax: 040558369 I

E-mail: Ifurlanis@units.it 

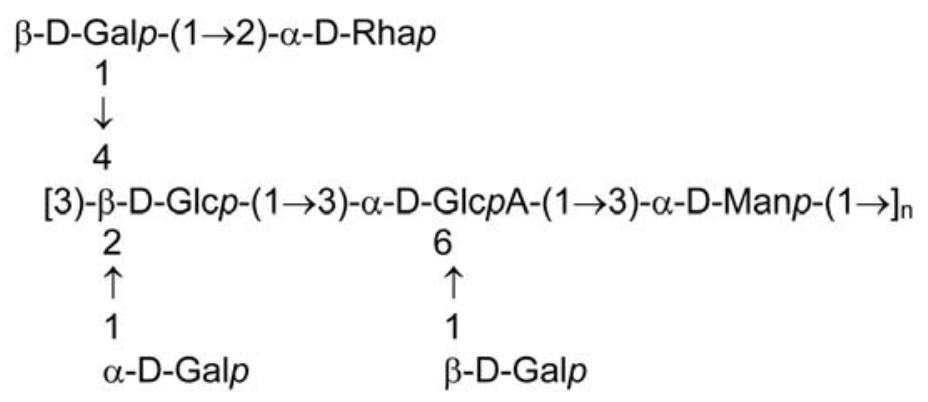

Figura I. Unità ripetitiva deacetilata dell'EPS cepaciano.

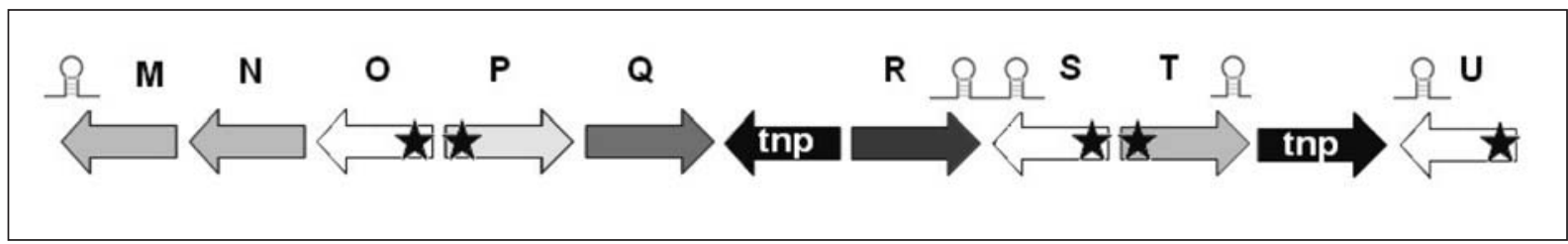

Figura II. Locus bcell di BTS2: in bianco le acetiltransferasi; in nero i geni codificanti per transposasi. Sono inoltre indicati i promotori (stelle) e le forcine di terminazione.

Tabella I. Quantificazione dei gruppi O-acetile presenti sull'esopolisaccaride cepaciano prodotto da tre specie batteriche diverse, ottenuta mediante spettroscopia ' $\mathrm{H}$ NMR. L'acetilazione viene espressa come quantità di acetili per unità ripetitiva polisaccaridica. Per il ceppo BTS7 il valore presentato è la media di I I preparazioni differenti; per i ceppi B383 e BTS2 il valore presentato è la media di 3 preparazioni differenti.

\begin{tabular}{cc}
\hline CAMPIONE & O-Ac/UR \\
\hline BTS7 & 3.0 \\
\hline B383-a & 2.8 \\
\hline BTS2-I & 2.2 \\
\hline
\end{tabular}

\section{BIBLIOGRAFIA}

1. Cescutti P, Impallomeni G, Garozzo D, Rizzo R. OAcetyl location on cepacian, the principal exopolysaccharide of Burkholderia cepacia complex bacteria. Carbohydr Res. 2011 Dec 27; 346 (18): 2905-12.

2. Ferreira AS, Leitão JH, Silva IN, et al. Distribution of cepacian biosynthesis genes among environmental and clinical Burkholderia strains and role of cepacian exopolysaccharide in resistance to stress conditions. Appl Environ Microbiol. 2010 Jan; 76 (2): 441-50. Epub 2009 Nov 30.

3. Moreira LM, Videira PA, Sousa SA, Leitão JH, Cunha MV, Sá-Correia I. Identification and physical organization of the gene cluster involved in the biosynthesis of Burkholderia cepacia complex exopolysaccharide. Biochem Biophys Res Commun. 2003 Dec 12; 312 (2): 323-33. 